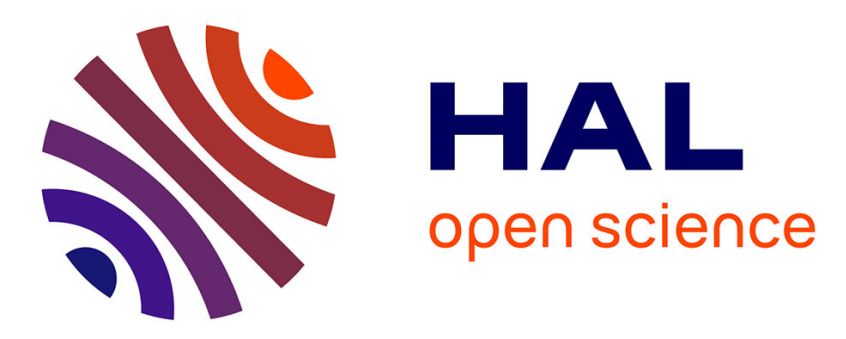

\title{
Modèle à constantes localisées de transducteurs : dissipation dans les couches limites
}

Pierrick Lotton, L. Husník, A.-M. Bruneau, M. Bruneau, Z. Škvor

\section{To cite this version:}

Pierrick Lotton, L. Husník, A.-M. Bruneau, M. Bruneau, Z. Škvor. Modèle à constantes localisées de transducteurs: dissipation dans les couches limites. Journal de Physique IV Proceedings, 1994, 04 (C5), pp.C5-217-C5-220. 10.1051/jp4:1994542 . jpa-00253037

\section{HAL Id: jpa-00253037 https://hal.science/jpa-00253037}

Submitted on 1 Jan 1994

HAL is a multi-disciplinary open access archive for the deposit and dissemination of scientific research documents, whether they are published or not. The documents may come from teaching and research institutions in France or abroad, or from public or private research centers.
L'archive ouverte pluridisciplinaire HAL, est destinée au dépôt et à la diffusion de documents scientifiques de niveau recherche, publiés ou non, émanant des établissements d'enseignement et de recherche français ou étrangers, des laboratoires publics ou privés. 


\title{
Modèle à constantes localisées de transducteurs : dissipation dans les couches limites
}

\author{
P. LOTTON, L. HUSNÍK*, A.-M. BRUNEAU, M. BRUNEAU et Z. ŠKVOR*
}

Laboratoire d'Acoustique de l'Université du Maine, URA 1101 du CNRS, Université du Maine, Avenue Olivier Messiaen, BP. 535, 72017 Le Mans cedex, France

* Laboratoire d'Acoustique de l'Université Technique de Prague, Université Technique de Prague, Technická, 16627 Praha 6, Czech Republic

\begin{abstract}
The main purpose of the paper is to provide, in an analytic way, an electrical network which describes the behaviour of electrostatic or electret transducers, and which takes into account the effects of the strong coupling between the mechanical and acoustical parts of the system; the conventional model seems to be improved to describe new miniaturized transducers with a good accuracy.
\end{abstract}

\section{INTRODUCTION.}

Les transducteurs acoustiques classiques contiennent, le plus souvent emprisonnés dans un petit volume clos, des fluides soumis à des mouvements alternatifs associés au mouvement imposé à une partie mécanique. Il en est ainsi par exemple pour les microphones, ou certains types de haut-parleurs, où le fluide forme une couche mince située entre un diaphragme et une paroi fixe, les mouvements de va-et-vient du fluide étant favorisés par la présence d'un volume d'expansion qui communique avec la couche de fluide par des orifices situés dans la paroi ou sur son pourtour.

Certains travaux proposent des calculs de caractéristiques de ce type de transducteurs, qui reposent sur les équations fondamentales de l'acoustique et leurs solutions précises [1]; ces calculs, dont les résultats reproduisent avec précision les résultats expérimentaux, restent d'usage délicat en raison de leur complexité et admettent au demeurant certaines hypothèses qui ne peuvent pas être retenues en toute circonstance.Le plus souvent, la modélisation de ce type de transducteurs fait appel à une représentation des divers éléments sous forme de circuits électriques équivalents, en particulier pour décrire le mouvement du fluide couplé à celui du diaphragme [2]. Mais là, les hypothèses sous-jacentes ne sont pas toujours exprimées, et les modèles ne sont pas directement déduits de la formulation de base des équations du problème.

\section{OBJECTIF.}

L'objet du présent travail est de proposer un modèle conduisant à un schéma équivalent reposant sur les équations fondamentales de l'acoustique et de la mécanique. La théorie utilisée prend ainsi en compte le fort couplage entre membrane et fluide; elle prend aussi en compte les effets de viscosité de cisaillement, due au fait que le fluide se déplace entre deux parois parallèles, ainsi que les effets de conduction de la chaleur qui ne sont pas totalement négligeables pour les transducteurs miniatures en hautes fréquences. Cette modélisation, appliquée ici au cas du microphone schématisé sur la figure 1, convient également pour décrire tout autre système comportant une mince couche de fluide emprisonnée entre une paroi vibrante et une paroi fixe, voire entre deux parois vibrantes [3], la très petite épaisseur de cette couche de fluide justifiant certaines hypothèses permettant de simplifier des équations de base du problème. 


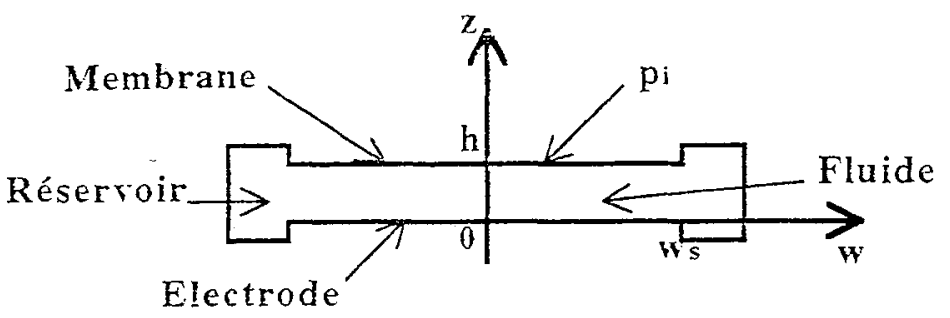

Figure $1:$ Le système microphonique étudié

\section{HYPOTHESES - EQUATIONS DU PROBLEME .}

\subsection{Les hypothèses}

La membrane, maintenue à sa périphérie, est excitée par une pression acoustique incidente $\mathrm{p}_{i}$ supposée uniforme sur sa surface. La position de cette membrane est repérée par la valeur de la coordonnée $z=h$, l'électrode arrière étant à la cote $z=0$. La coordonnée généralisée orthogonale à $z$ est notée $w(w=(x, y)$ ou $\mathbf{w}=(r, \theta)$ suivant la géométrie du système), et l'entrée du volume d'expansion est caractérisée par $\mathbf{w}=\mathbf{w}_{\mathbf{S}}$.

Du fait de la faible valeur $\mathrm{h}$ de l'épaisseur du fluide, la pression p peut être supposée constante sur cette épaisseur et par suite ne dépendre que de la variable $w: p=p(w)$. Dès lors la composante suivant $z$ de la vitesse particulaire doit être négligée : $v_{z}=0$ et $v_{\mathbf{w}}=\mathbf{v}(\mathbf{w}, z)$. Enfin la prise en compte des effets de la viscosité de cisaillement conduit à négliger les variations des grandeurs avec la coordonnée $\mathbf{w}$ devant les variarions avec la coordonnée $z:\left|\operatorname{grad}_{\mathbf{w}}\right| \ll\left|\partial / \partial_{z}\right|$.

\subsection{Equations du mouvement du fluide}

Compte tenu des hypothèses faites, la description du problème repose sur les équations suivantes :

- l'équation de Stokes-Navier, qui se réduit à la loi de Poiseuille pour les fluides compressibles

$$
\left(\partial_{c t}{ }^{-1}{ }^{\prime} \partial^{2} z z\right) v(w, z)=-(\rho c)^{-1} \operatorname{grad}_{w} p(w)
$$

où la longueur caractéristique $1_{\mathrm{v}}^{\mathrm{V}}=\mu /(\rho \mathrm{c})$, c représentant la vitesse adiabatique du son, $\mu$ le coefficient de viscosité de cisaillement et $\rho$ la masse volumique de l'air,

- l'équation de conservation de la masse, qui s'écrit pour une couche de fluide dont l'épaisseur $h$ est considérée comme un élement différentiel du 1 er ordre

$$
\left.h \operatorname{div}_{\mathbf{w}}<\mathbf{v}(\mathbf{w}, z)\right\rangle+\mathrm{i} \omega \xi(\mathbf{w})=-(i \omega / \rho) h\left\langle\rho^{\prime}(\mathbf{w}, z)\right\rangle,
$$

où $<\bullet>$ représente la valeur moyenne sur l'épaisseur du fluide d'une grandeur, et $\xi(w)$ le déplacement de la membrane,

- l'équation d'état, reliant la variation de masse volumique $\rho^{\prime}$ aux deux variables indépendantes $p$ variation de pression et $\tau$ variation de température

$$
\left\langle\rho^{\prime}(w, z)>=\left(\gamma / c^{2}\right)[p(w)-\beta<\tau(w, z)>],\right.
$$

où $\gamma=C_{\mathrm{p}} / \mathrm{C}_{\mathrm{v}}$ est le rapport des capacités calorifiques à pression et volume constant et $\beta=(\partial \mathrm{P} / \partial \mathrm{T})_{\rho}$ l'augmentation de pression isochore,

-l'équation de Fourier pour la conduction de la chaleur

$$
\left(\partial_{c t}-l_{h} \partial_{z z}^{2} \tau(w, z)=[(\gamma-1) /(\beta \gamma)] \partial_{c t} p(w),\right.
$$

où la longueur caractéristique $\mathrm{l}_{\mathrm{h}}=\lambda_{\mathrm{h}} /\left(\rho \mathrm{c} \mathrm{C}_{\mathrm{p}}\right), \lambda_{\mathrm{h}}$ représentant la conductivité thermique . 


\subsection{Conditions aux frontières}

Les conditions sur les parois $z=0$ et $z=h$ assurent la continuité des vitesses et des flux de chaleur et, à la périphérie, la variation de pression et la vitesse particulaire sont reliées par une condition d'impédance.Ces conditions s'écrivent :

$$
\begin{aligned}
& \tau(\mathbf{w}, 0)=\tau(\mathbf{w}, \mathrm{h})=0 \\
& \mathbf{v}(\mathbf{w}, 0)=\mathbf{v}(\mathbf{w}, \mathrm{h})=0 \\
& p\left(\mathbf{w}_{\mathbf{s}}\right)=Z<\mathrm{v}\left(\mathbf{w}_{\mathbf{s}}, \mathrm{z}\right)>
\end{aligned}
$$

\subsection{Equations régissant le mouvement de la membrane}

Le déplacement $\xi$ de la membrane dont les faces sont soumises aux pressions $p_{i}$ et $p(w)$ est régi par l'équation :

$$
\mathrm{T}\left(\Delta_{\mathbf{w}}+\mathrm{K}^{2}\right) \xi(\mathbf{w})=\mathrm{p}_{\mathrm{i}}-\mathrm{p}(\mathbf{w}),
$$

et la condition à la périphérie s'écrit :

$$
\xi\left(w_{\mathbf{s}}\right)=0
$$

où $K^{2}=\omega^{2} \mu_{\mathrm{S}} / \mathrm{T}$, T étant la tension de la membrane et $\mu_{\mathrm{S}}$ sa masse surfacique.

\section{SOLUTION DU PROBLEME.}

Le système d'équation (1) à (6) permet d'obtenir l'équation de propagation suivante pour la pression:

$$
\left(\Delta_{\mathbf{w}}+\chi^{2}\right) \mathrm{p}(\mathbf{w})=\zeta \xi(\mathbf{w})
$$

avec

$$
\chi^{2}=\left(\omega^{2} / \mathrm{c}^{2}\right) \gamma\left[1-[(\gamma-1) / \gamma] \mathrm{F}_{\mathrm{h}}\right] / \mathrm{F}_{\mathrm{v}} \quad \text { et } \quad \zeta=-\rho \omega^{2} /\left(\mathrm{h} \mathrm{F}_{\mathrm{V}}\right)
$$

où

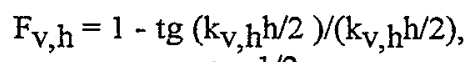

$k_{\mathrm{V}}=(-\mathrm{i} \omega \rho / \mu)^{1 / 2}$ et $\mathrm{k}_{\mathrm{h}}=\left(-\mathrm{i} \omega \rho \mathrm{C}_{\mathrm{p}} / \lambda_{\mathrm{h}}\right)^{1 / 2}$ représentant les nombres d'onde associés respectivement aux mouvements tourbillonnaire et entropique :

Les solutions du problème global sont alors données par la résolution des équations couplées (8) et (10) en prenant en compte les équations aux limites (7) et (9). L'expression du déplacement $\xi(w)$ de la membrane est cherché sous forme d'un développement sur les fonctions propres $\psi_{\mathrm{n}}\left(\mathrm{k}_{\mathbf{w n}} \mathbf{w}\right)$ solutions du problème aux valeurs propres associé aux équations (8)et (9) :

avec

$$
\begin{aligned}
& \xi(w)=\sum \xi_{n} \psi_{n}\left(k_{w n} w\right) \\
& \xi_{n}=T^{-1}\left[K^{2}-\left(k_{w n}\right)^{2}\right]^{-1} \iint d w_{0}\left[p_{i}-p\left(w_{0}\right)\right] \psi_{n}\left(k_{w n} w_{0}\right)
\end{aligned}
$$

Alors, la solution de l'équation (10) gouvernant la variation de pression du fluide peut s'écrire:

$$
\mathrm{p}(\mathbf{w})=\sum \mathrm{p}_{\mathrm{n}} \psi_{\mathrm{n}}\left(\mathrm{k}_{\mathbf{w n}} \mathbf{w}\right)+\Phi(\chi \mathbf{w})
$$

avec

$$
\mathrm{p}_{\mathrm{n}}=\zeta \xi_{\mathrm{n}}\left[\chi^{2}-\left(\mathrm{k}_{\mathbf{w n}}\right)^{2}\right]^{-1}
$$

et où $\Phi(\chi w)$ est solution de l'équation homogène associée à cette équation (10).

La condition d'impédance à la périphérie (éq.7) permet de déterminer complètement la fonction $\Phi(\chi w)$. Finalement, $\xi_{n}$ peut s'écrire :

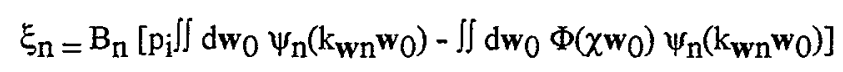

avec

$$
B_{n}=\left\{T\left[K^{2}-\left(k_{w n}\right)^{2}\right]+\zeta\left[\chi^{2}-\left(k_{w n}\right)^{2}\right]^{-1}\right\}^{-1} \text {. }
$$




\section{SCHEMA ELECTRIQUE EQUTVALENT.}

L'expression du déplacement $\xi(w)$ (éq. 11 et 13) permet alors d'obtenir l'impédance $Z_{0}$ du circuit électrique équivalent, rapport de la pression $p_{i}$ au débit $U=i \omega S<\xi>$, $S$ étant la surface du diaphragme. L'impédance obtenue conduit au schéma équivalent donné par la figure $2 \mathrm{a}$, dans lequel la $\mathrm{n}$ ème branche $\left(Z_{n}\right.$, figure $2 b)$ correspond au nème mode propre $\xi_{n} \Psi_{n}\left(k_{w n} w\right)$ de vibration de la membrane.

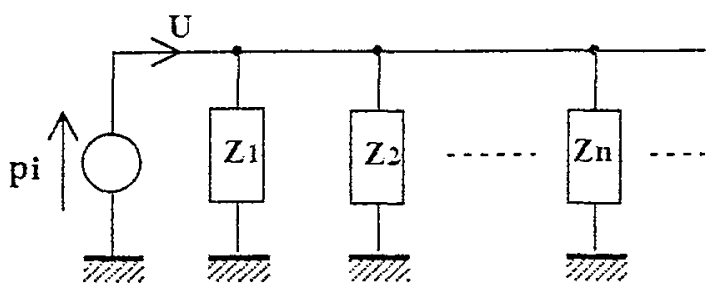

(a)

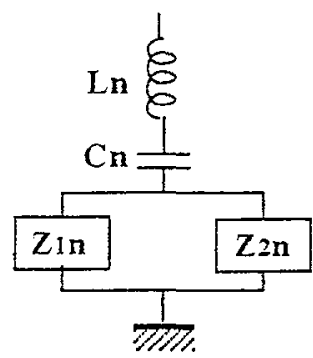

(b)

Figure 2 : Circuit électrique équivalent.

a- Structure générale du circuit.

b- Schéma de la nème branche $Z_{n}$.

Les éléments du circuit, calculés pour une géométrie cylindrique, ont la forme suivante :

$$
\begin{array}{lll}
\mathrm{L}_{\mathrm{n}}=\left(\mathrm{k}_{\mathrm{wn}}\right)^{2} \mu_{\mathrm{s}} /\left(4 \pi \mathrm{N}_{\mathrm{n}}\right), & & \mathrm{C}_{\mathrm{n}}=4 \pi \mathrm{N}_{\mathrm{n}} /\left[\left(\mathrm{k}_{\mathrm{wn}}\right)^{4} \mathrm{~T}\right], \\
\mathrm{Z}_{1 \mathrm{n}}=\zeta /\left(\mathrm{i} 4 \pi \omega \mathrm{N}_{\mathrm{n}}\right), & \text { et } & \mathrm{Z}_{2 \mathrm{n}}=\mathrm{i} \zeta\left(\mathrm{k}_{\mathrm{wn}}\right)^{2} /\left(4 \pi \omega \chi^{2} \mathrm{~N}_{\mathrm{n}}\right) .
\end{array}
$$

$L_{n}$ est $C_{n}$ comportent les termes d'inertie et de compliance de la membrane, $Z_{1 n}$ et $Z_{2 n}$ comportent ceux représentant l'impédance de la lame de fluide. Tous ces termes incluent également un facteur $N_{n}$ qui traduit l'effet de la réaction du volume périphérique à linterface membrane-fluide. Pour un volume infini correspondant à une condition de Dirichlet ( $Z=0$, relation 7 ), $\mathrm{N}_{\mathrm{n}}=1$.

On note que le schéma équivalent obtenu diffère notablement de ceux qui sont d'usage courant. En particulier, la contribution de chacun des modes (correspondant au nombre quantique $n$ ) prend en compte le système dans son ensemble, membrane couplée à la lame de fluide et au volume périphérique, alors que dans les circuits classiques[2], l'effet des modes supérieurs se réduit à celui des modes "in vacuo" de la membrane.

L'analyse et l'exploitation de ces résultats sont en cours.

[1] ZUCKERWAR A.J, Theoretical Response of Condenser Microphones, J.Acoust.Soc.Am., 64, (5), (1978), 1278-1285.

[2] SKVOR Z., Vibrating Systems and their Equivalent Circuits, (Elsevier Science Publishers, Amsterdam, Oxford, New York, Tokyo, 1991).

[3] ŠKVOR Z., KADLEC F., BRUNEAU A.M., LOTTON P., HUSNÍK L., NOVÁK A., "Piezoelectrical Transducer with Piezopolymer Diaphragm", C.T.U. PRAGUE, Workshop 93, Prague Janvier 1993, pp 193-194. 